\title{
Salidas de campo EN LA FORMACIÓN DE EMPRENDEDORES
}

\section{Resumen}

Este artículo de Investigación hace parte de los resultados del proyecto "Pedagogía y didáctica de las humanidades" desarrollado por los autores para la Vicerrectoría de Investigaciones de la Universidad EAN.

El proyecto tiene como objetivo preparar salidas de campo como estrategia pedagógica.

En este orden de ideas, busca identificar y unificar los elementos que conforman la estructura de la salida de campo, permitiendo planificar e implementar un tipo de práctica que fortalezca las competencias transversales, así como plantear un modelo de indicadores para su evaluación.

\section{Palabras clave}

Salidas de campo

Estrategia pedagógica Emprendedores

\author{
Alexander CelyRodríguez* \\ Nélson Díaz Cáceres** \\ David Ricardo Ocampo Eljaiek $k^{* * *}$
}

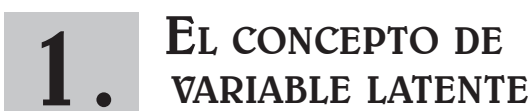

La metodología de creación de ambientes y experiencias que afiancen el compromiso y el conocimiento de los estudiantes, es la base de la propuesta pedagógica de cada universidad; que consiste en esencia, en generar espacios motivadores e interesantes para el aprendizaje.

Teniendo en cuenta lo anterior, se ha recurrido a la salida de campo como estrategia pedagógica, que brinda al estudiante una visión real y directa del entorno y un contacto concreto con el mundo donde desarrolla su laborpráctica.

Una salida puede mostrar análisis de los fenómenos de la realidad, de manera más compleja de lo que se logra en horas de

* Profesor de la Universidad Pedagógica Nacional, Catedrático de la Universidad EAN e Integrante de Grupo Compedean.

** Profesor Asociado de la Universidad EAN, Integrante del Grupo Compedean.

*** Profesor Catedrático de la Universidad EAN, Integrante del Grupo Compedean.

Este artículo fué entregado el 4 de agosto de 2008 y su publicación aprobada por el Comité Editorial el 16 de agosto de 2008. 
clase. Por lo tanto esta investigación pretende mostrar cómo las salidas de campo tienen en su estructura fundamentos de aprendizaje significativo "se aprende una vez y nunca se olvida" (Ausubel ,1970).

Aquí se realiza un análisis de la importancia de las salidas de campo desde la educación, la pedagogía y la didáctica, analizadas además desde la formación de competencias sociohumanísticas relacionadas tambiéncon la comprensión del entorno geográfico como forma de aprender y de enseñar, visualizando por últimoel contexto de la formación de emprendedores.

Se espera que el análisis realizado en esta investigación ayude a profesores y estudiantes, en el diseño y realización de esta estrategia que a su vez, fortalece el proceso de formación de emprendedores.

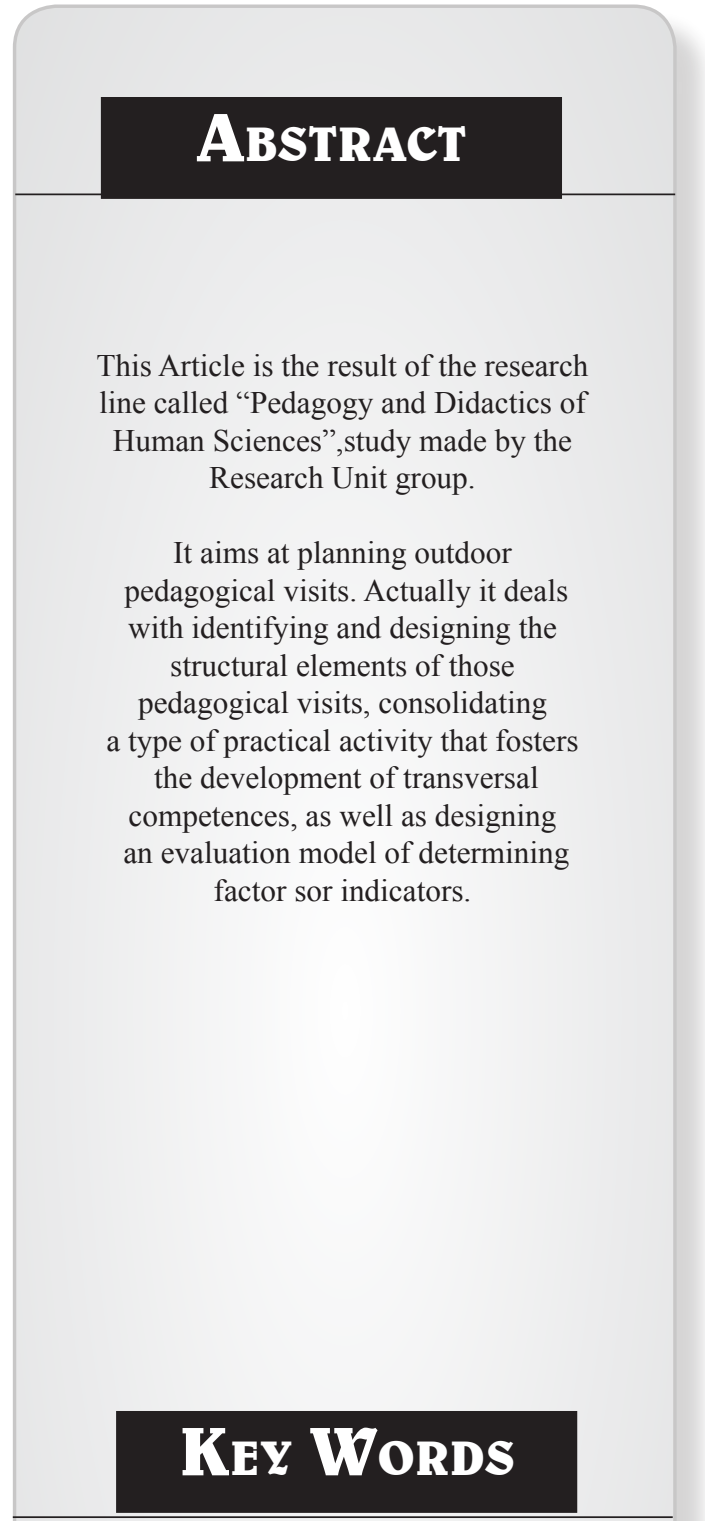

Outdoort pedagogical visits Pedagogical strategies Entrepreneurs. 


\section{Pedagogía y didáctica de las humanidades}

Con el fin de sistematizar las diferentes estrategias pedagógicas y didácticas que se desarrollan dentro y fuera del aula en el proceso de enseñanza y aprendizaje de las humanidades; en este proyecto de investigación se realiza una revisión teórica al proceso de enseñanza y aprendizaje en el marco del enfoque constructivista; modelo propuesto por el Proyecto Educativo Institucional de la Universidad.

La teoría entorno a la educación presenta diversos momentos, tendencias, estilos y modelos pedagógicos, los cuales han determinado a lo largo del tiempo, su función y actuación en las escuelas; su efecto en los estudiantes y por lo tanto en las sociedades.

Frentealosmodelospedagógicostradicionales, el constructivismo representa una alternativa que posibilita el cuestionamiento de las relaciones que ocurren entre aprendizaje y estudiante, dándole a éste último un papel protagónico, generando una dinámica diferente en la que el estudiante es actor y no un ente pasivo de la enseñanza.

En Colombia se ha desatado una fuerte tendencia a trabajar desde las corrientes constructivistas, en la medida en que estas promueven y posibilitan el desarrollo de programas que vinculan el trabajo en el aula con sus proyecciones en ámbitos exteriores. Bajo el lente constructivista, el aprendizaje debe estar siempre asociado al desarrollo del ser humano.
Lo que plantea el constructivismo pedagógico es que el verdadero aprendizaje humano es una construcción de cada alumno que logra modificar en su estructura mental, y alcanzar un mayor nivel de diversidad, de complejidad y de integración. Es decir, el verdadero aprendizaje es aquel que contribuye al desarrollo de la persona....el desarrollo del individuo en formación es el proceso esencial y global, en función del cual se puede explicar y valorar cada aprendizaje particular (Flórez,1994:235).

Todos los movimientos pedagógicos y las inquietudes desatadas en los últimos años, apuntan a dinamizar el proceso de enseñanza a partir de la instauración del aprendizaje significativo. Este se apoya en la generación de espacios de debate, marcos de cuestionamiento y controversia que de una u otra manera, logran romper con esquemas y tradiciones en la educación.

El aprendizaje se da través de la educación. El concepto educación, comprende el desarrollo de acciones sociales mediante las cuales los seres humanos intentan promover de alguna manera, la personalidad de otros seres humanos. En las instituciones educativas se da un aprendizaje social y un aprendizaje técnico, replicando experiencias reales que facilitan el aprendizaje. Más estas experiencias, no pueden desvincularse del fin superior de la formación de la personalidad en el contexto de la experiencia y la reflexión social. 
En cuanto a lo que hace al aprendizaje coexisten varias posturas y fundamentos relacionados con el mismo. Son muy diversos los elementos que interactúan en el proceso de aprendizaje, entre ellos los procesos de enculturación, socialización, educación y aprendizaje social.

Para el caso, queremos señalar que el denominado aprendizaje significativo busca desde la relevancia del conocimiento, alcanzar una interiorización y apropiación de saberes en los estudiantes convirtiéndose en un verdadero aprendizaje "en y para la vida". Sólo a partir de la práctica de la vida, de su funcionalidad y su reflexión, el nuevo aprendizaje no será arbitrario sino por el contrario sustancialmente significativo.

"La significatividad del aprendizaje se refiere a la posibilidad de establecer vínculos sustantivos y no arbitrarios entre lo que hay que aprender -el nuevo contenido- $y$ lo que ya se sabe, lo que se encuentra en la estructura cognitiva de la persona que aprende -sus conocimientos previos. Aprender significativamente quiere decir poder atribuir significado al material objeto de aprendizaje (Coll, 2002)".

Antonio Moreira (2000) afirma que "la enseñanza y el aprendizaje serían altamente ineficientes si el estudiante tuviese que redescubrir los contenidos para que el aprendizaje fuese significativo". El aprendizaje significativo requiere de permanente comunicación entre el docente y el estudiante. Es un proceso de construcción conjunta entre ambos agentes (docente-estudiante), un proceso orientado a compartir universos de significación cada vez más amplios y complejos; en éste el profesor intenta que las construcciones de los estudiantes se aproximen progresivamente a las que se consideren correctas y adecuadas para comprender la realidad. La enseñanza y la intervención del profesor constituyen una ayuda, durante el tiempo en que el estudiante procede a la construcción.

Un elemento fundamental dentro del aprendizaje es la motivación, factor clave a la hora de abordar nuevos aprendizajes en profundidad y de establecer relaciones y vínculos entre lo que ya sabe y lo que debe aprender el estudiante. En el aspecto motivacional hay que tener en cuenta las distancias; un aprendizaje será más o menos significativo mientras la distancia entre el estudiante y el conocimiento sea razonable, es decir, no tan complejo que sea casi imposible adquirirlo o tan familiar que sea muy fácil obtenerlo.

Otro aspecto en cuanto al proceso enseñanza y aprendizaje y la función de las salidas pedagógicas es la relación que establece el profesor con sus estudiantes. Una buena relación y una excelente comunicación permitirán que los logros propuestos se alcancen con menos dificultades. La aceptación, la confianza mutua y el respeto; posibilitan las relaciones afectuosas, brindan seguridad y facilitan la formación de una autoimagen ajustada y positiva (Coll, 2002).

Para que el aprendizaje de los estudiantes sea lo más significativo posible, supone establecer condiciones que permitan una enseñanza individualizada, que haga énfasis en distintos factores como la actuación y la actividad mental del estudiante; esto hace del profesor un profesional insustituible e irremplazable.

Al hablar de aprendizaje se pueden establecer diversas formas de lograrlo y diferentes 
manifestaciones en el proceso de adquisición. El aprendizaje social, corresponde a los procesos de aprendizaje en los cuales se investiga y se reflexiona en condiciones organizadas y conjuntamente con el apoyo de los contenidos técnicos de importancia social como valores, expectativas, normas, roles, reglas y comportamientos.

De igual forma en el aprendizaje se incluyen tres procesos que son simultáneos: la adquisición de información nueva (con la que se está de acuerdo, se contradice o sustituye); la transformación (se trata la información para superarla o adaptarla a nuevas tareas) y la evaluación o comprobación de si nuestra preparación de la información es adecuada a la nueva aplicación (Bruner, 1970). El aprendizaje es central en la pedagogía sin embargo, no debe confundirse con la educación.

En el proceso de aprendizaje es fundamental articular, entre otros, diferentes factores biológicos, culturales, emocionales y sociales. En el aspecto biológico el desarrollo de los estudiantes, las condiciones ambientales y los elementos constitutivos de cada etapa de crecimiento, posibilitarán en alguna forma la instauración de un proceso de aprendizaje.

Biológicamente en el proceso de aprendizaje existen dos variables importantes que lo fundamentan, la concentración y la atención. "El propósito de la concentración es adquirir la sensación de precisión en la ejecución de la actividad, la próxima vez que desee aprender algo nuevo, entréguese a la obtención del sentimiento de precisión adecuado para usted. Esto le permitirá ejecutar la acción y experimentar con intensidad la emoción de aprender, para que después realice un análisis crítico y evalúe los resultados alcanzados durante el aprendizaje". "La dificultad para concentrarse y fijar la atención y, por lo tanto, para mejorar los procesos de aprendizaje se origina en la forma como opera el cerebro. Este funciona en cuatro frecuencias diferentes... el aprendizaje de nuevos conocimientos se logra cuando la persona sabe crear un estado de gran receptividad para recibir con mayor facilidad los contenidos conceptuales y así comprenderlos con profundidad, asimilarlos e integrarlos, logrando una mejor retención y el dominio de los conocimientos, en forma autónoma". (Argüelles y Nagles, 2002).

De igual forma en el aprendizaje influyen los elementos sociales, culturales y emocionales los cuales cumplen con una función importante dentro de este proceso y se entrelazan para consolidarlo. De ahí la importancia de resaltar el papel del lenguaje, sus diversas manifestaciones y la forma como éste determina un aprendizaje.

"El lenguaje de la educación, si ha de ser una invitación a la reflexión y a la creación de cultura no puede ser el denominado lenguaje incontaminado de la realidad y la 'objetividad'. Debe expresar las actitudes e invitar a la contra actitud y en ese proceso dejar margen para la reflexión, para la metacognición. Es esto lo que nos permite acceder a un estrato superior, proceso que permite objetivar a través del lenguaje 0 de imágenes lo que hemos pensado y luego reflexionar sobre ello y reconsiderarlo (Bruner, 1970: 134)".

En el aspecto cultural el lenguaje debe permitir evidenciar la articulación entre la lectura y el lenguaje simbólico, siendo complementado por el aspecto emocional, que dará paso al juego de intereses y motivaciones existentes en un proceso de aprendizaje. 
En la perspectiva cognitiva la enseñanza parte de problemas o temas de interés vital sentidos por los alumnos y que hacen parte de su vida real. El mismo aprendiz alimenta el proceso de pensar y aprender con sus conocimientos, ideas y experiencias previas acerca del problema propuesto. La colaboración, la búsqueda de conjeturas, pruebas y argumentaciones, deben ser propuestas por los mismos alumnos como participantes activos y creadores, en cooperación con sus compañeros.

Laalegríaylasatisfacciónobtenidadelaprendizaje brindan la confianza necesaria para la búsqueda de nuevos conocimientos; sin embargo el desequilibrio generado por la insatisfacción y la duda que resulta del conflicto cognitivo, crea una especie de ruido, de impotencia, de vacío, de contradicción o perplejidad ante lo desconocido. Cuando no hay congruencia entre lo que el alumno sabe o cree saber y la complejidad del fenómeno que tiene que abordar, sobrevienen la inseguridad, la incertidumbre y la búsqueda de un nuevo equilibrio cognitivo, que no terminará ni hallará compensación sino cuando ocurra la nueva comprensión congruente, que permita la aceptación y el compromiso del juicio del aprendiz. En consecuencia, la motivación es intrínseca al aprendizaje mismo y no puede marginarse ni aislarse cuando el profesor evalúa, ni cuando el alumno se autoevalúa (Flórez, 1999).

El aprendizaje solo es real y significativo cuando aprender posibilita la creación de saberes y la aplicación de esos saberes en la práctica cotidiana.

Los profesores al enseñar pretenden que los alumnos aprendan a conocer el mundo en que se vive. En múltiples ocasiones se desaniman al comprobar que los jóvenes no comparten sus ilusiones relacionadas con las explicaciones rigurosas de los hechos sociales.

La preocupación es mayor, debido a que en los espacios académicos de humanidades y de formación integral se estudian precisamente los mecanismos que rigen los fenómenos sociales y las interacciones entre los seres humanos y su medio. Es por esto que crear un ambiente de comunicación didáctica es, en realidad, un reto constante y los obstáculos que dificultan una buena enseñanza son muchos. Entonces, es necesario priorizar de manera franca y directa a aquellos que afectan al aprendizaje del alumno, más cuando nuestra meta radica en formar individuos autónomos para una sociedad superinformada, plural y democrática (Souto, 1999). 
Hacecasiunadécadaeltérminocompetencias, más claramente el modelo de educación basado en el desarrollo de competencias, viene siendo usado con relevancia en la política educativa colombiana, bien sea como resultado de un proceso sustancial o como un fenómeno o moda intelectual.

La confusión y el desconcierto son comunes entre los educadores, porque competencia no es un concepto originario de la pedagogía y tampoco ha hecho parte de la reflexión que realizaban los educadores.

La evaluación de competencias adoptada por el ICFES representa un cambio cualitativo en la manera de entender y practicar la evaluación de calidad, siendo la nueva manera de concebir la forma de hacer bien las cosas. Las implicaciones en el acto educativo son de índole variada, debido a los cambios realizados en el objeto de la evolución. Ello implica adoptar modificaciones en los propósitos, los contenidos y los métodos de aprendizaje, debido a que la evaluación se halla conectada en línea directa con la competencia de la acción educativa.

Es necesario aclarar que la evaluación por competencias, no es una técnica para hacer exámenes que puede ser adoptada por los los docentes y usada de manera esporádica o permanente sin afectar otros componentes básicos y específicos de los distintos saberes académicos.

Se entiende por competencia "la capacidad para hacer uso creativo de los conocimientos adquiridos en la escuela y fuera de ella". De acuerdo con el equipo de competencias de la Universidad Nacional de Colombia, este concepto se utiliza para enfatizar que el propósito de la educación no es lograr que el estudiante memorice un sinfín de conocimientos e información; sino que desarrolle sus potencialidades y capacidades y haga uso creativo de los saberes y herramientas que la escuela brinda. De ahí que el concepto de competencia se entienda como el conocimiento perfecto e ideal al que todos debemos llegar.

Cuando se habla del desarrollo de competencias en los educandos, se hace referencia a formar estudiantes capaces de hacer uso adecuado de los contenidos o saberes de la ciencia, de sus métodos de trabajo, de sus símbolos y estilos de comunicación. En otras palabras, se trata de desarrollar en los estudiantes la capacidad de análisis y de lectura de la realidad social y cultural, de establecer relaciones entre fenómenos y conceptos, de formular hipótesis, de argumentar estas hipótesis e interpretaciones y de comunicarlas de manera clara.

Enelcasodel procesoenseñanza-aprendizaje de las humanidades se hace referencia a las competencias transversales desde el contexto de la autonomía del aprendizaje y de las mismas destrezas cognitivas, para el desarrollo de cualquier carrera universitaria. La transversalidad da coherencia al currículo incursionando en las unidades de estudio para la realización de trabajos comunes, con un grado de especificidad en la carrera 
seleccionada y haciendo que el estudiante tome decisiones ante situaciones concretas y donde debe aplicar sus conocimientos. Los actores en la transversalidad desarrollan diversos tópicos, así como didácticas que se vuelven un conjunto de métodos, estrategias y recursos utilizados en la enseñanza de las Ciencias Sociales.

Las humanidades afectan el conjunto de comportamientos socio-afectivos, desarrollando una tarea específica en cada individuo donde buscan situarlo en un contexto de tiempo y espacio para la resolución de problemas en el mundo contemporáneo laboral; se relacionan con otros seres que también persiguen su propia autonomía no sólo desde la formación básica, sino en cuanto a los referentes sociales y culturales; desarrollan además, una comprensión global del mundo, desde un enfoque multicultural y propician el desarrollo integral de la realidad nacional e internacional.

La educación basada en competencias desde el ámbito socio-humanístico integra diversos conceptos: habilidades, conocimiento, destrezas, actitudes, valores; generando nuevas alternativas a los problemas de nuestra sociedad, en donde el estudiante realiza consensos de acuedo con el grado de complejidad deseado y a su vez de pertenencia con respecto a la cultura.

Los currículos flexibles facilitan el saberhacer desde el ámbito sociocultural y articulan problemas con posibles soluciones que relacionan el contexto educativo con el contexto pedagógico inherente a cada individuo. El diseño de estrategias debe favorecer la concepción del aprendizaje y su relevancia. En este mismo sentido, la concepción del emprendimiento no sólo atañe a los nuevos profesionales, sino a las estrategias propuestas por los docentes desde su unidad académica. El currículo no debe ser algo construido de antemano sino un propósito común de construcción permanente en la práctica diaria, de modo que permita transformar, desarrollar y respetar la formación integral de los educandos en un modelo pedagógico flexible.

Como bien lo plantea Silva (2003), el conocimiento no es la ventaja competitiva, porque se requiere su transformación en productos y servicios; estos a su vez se desarrollan mediante estrategias de relación psico-social entre los docentes y estudiantes. Como afirma lanfrancesco (1998) "el principal papel que debe cumplir el currículo es el de favorecer el desarrollo humano y social".

Las actividades materializadas en didácticas, pedagogías y estrategias, desde el concepto sociohumanístico contribuyen en el contexto de aprendizaje de los individuos, en procesos de formación y madurez personal. Aportan también fundamentos antropológicos, axiológicos, sociológicos, psicológicos, epistemológicos y pedagógicos;evidenciados no sólo en la competencia global, sino en cada una de las que se desglosa la unidad de estudio. Forman un ciudadano comprometido con la transformación de la sociedad y el desarrollo del país de una manera responsable, crítica, creativa y solidaria. Fortalecen la formación integral y permiten el desarrollo de las personas.

Al igual que el concepto de competencias, las humanidades entrelazan saberes de distintas áreas en caminos transversales y en procesos de crecimiento que coadyuvan a la integración de las personas en los procesos continuos de aprendizaje, autonomía y trabajo intelectual. 


\section{LA SALIDA DE CAMPO Y LA COMPRENSIÓN DEL ENTORNO GEOGRÁFICO: HACIA LA FORMACIÓN DE NUEVAS ESPECIALIDADES}

Existen cada vez más desafíos para la comprensión del espacio geográfico. En la relación espacio-tiempo-empresa este es denominado entorno geográfico. La enseñanza del entorno geográfico, principalmente en función de las dificultades que el mismo plantea desde una perspectiva socio-espacial, se presenta de una forma bastante compleja; mucho más cuando se pretende promover una visión articulada de los hechos y una "forma de expresión de la aplicabilidad del raciocinio espacial" (Ausubel, 1970).

Al respecto, es necesario revisar la manera de enseñar el entorno geográfico a partir del trabajo con conceptos y contenidos geográficos. Sin embargo este asunto ha sido tratado en el ámbito educativo con preocupante negligencia, lo cual es mucho más grave cuando nos encontramos frente a rápidas y muy significativas transformaciones en todos los sectores de la vida contemporánea a causa de la Tercera Revolución Industrial o Tecnológica. En tanto estas innovaciones tecnológicas afectan las sociedades de una manera desigual, acentúan las diferencias de comprensión del desarrollo socio-espacial.

La enseñanza de las ciencias sociales y las humanidades en general ha pasado por distintos procesos y concepciones teóricas, que han introducido nuevas orientaciones metodológicas para cada área de estudio. Uno de los objetivos centrales que rige este campo de estudio, no obstante, los cambios y las perspectivas metodológicas, es el de propiciar en los estudiantes conciencia entorno a la realidad social y espacial. En otros términos, que el joven comprenda su papel como agente de transformación social y que no se conciba de manera simple como un elemento dislocado de la realidad a la que pertenece.

Enseñar a interpretar y comprender el entorno geográfico, juega un papel relevante en la medida en que despierta en el estudiante la conciencia del descubrimiento y de la percepción socio-espacial, propiciando la capacidad de contemplar de una manera crítica y consciente cada uno de los acontecimientos de la vida cotidiana.

La enseñanza de las ciencias sociales y las humanidades no se limita a la simple transmisión de conocimientos; debe por el contrario, contribuir a mejorar el análisis de la comprensión de la realidad. En este sentido es necesario mejorar las herramientas y las técnicas de enseñanza, utilizando diversos recursos didácticos y desarrollando instrumentos que faciliten un nivel de comprensión que encauce la intervención en la sociedad y la actitud hacia el entorno.

Los recursos didácticos son los medios que el profesor utiliza para facilitar el aprendizaje. Son aquellos elementos y procedimientos que permiten alcanzar los objetivos de una clase, de un conjunto de clases o de todo un curso. La didáctica atañe igualmente a la organización de la clase, al uso de materiales, al desarrollo de actividades y al proceso de evaluación. 
La identificación de los recursos didácticos apropiados al inicio de un determinado proyecto debe tener en cuenta, entre otros aspectos: los objetivos, las necesidades o los requerimientos dados para el proceso; así mismo debe incluir el modelo didáctico y el mismo concepto de aprendizaje dentro del cual se enmarque la tarea pedagógica; finalmente, la adaptación necesaria para acercar las condiciones ideales a las reales. Una vez identificados estos factores, los recursos didácticos deben propiciar la dinámica pedagógica, la participación, la integración y la cohesión del grupo; estimular la motivación y el interés de los estudiantes, ser coherentes frente a las diferencias individuales, enriquecer las experiencias, fomentar la creatividad de los estudiantes y fortalecer la política de flexibilidad del programa; de modo que éste se adapte a nuevas situaciones y a diferentes configuraciones grupales.

\subsection{Lasalidadecampo:contribuciones y acercamientos teóricos}

La salida de campo no solamente es un método de investigación, sino es una excelente estrategia pedagógica y un gran recurso didáctico. Puede concebirse en el ámbito de las ciencias sociales y por tanto de las humanidades, "como un instrumento de análisis espacial que permite el reconocimiento del objeto y que, siendo parte de un método de investigación, permite la inserción del investigador en conjunto" en el movimiento de la sociedad (Suertegaray, 2002).

Distintos son los aportes de la salida de campo para el desarrollo de la percepción socio-espacial de la realidad. En un primer momento, se toma como un elemento fundamental para los trabajos de estudio del medio, es decir en actividades que buscan confrontar al estudiante con una realidad específica, donde participan profesores y estudiantes. En segundo lugar, el trabajo de campo lleva a la práctica de leer el paisaje, tan importante en el desarrollo de cualquier investigación de las ciencias sociales. En este mismo sentido, Thralls afirma que la salida de campo:

- "Permite la posibilidad de aprender directamente. El estudio de la realidad se lleva a cabo colocando al estudiante como agente activo del proceso enseñanzaaprendizaje. El mismo descubre los hechos, obtiene impresiones, desarrolla conceptos y percibe la interrelación del hombre con su medio.

- Estimula el espíritu de exploración, mientras agudiza el deseo por la investigación.

- Despliega la imaginación, mientras activa la motivación y el deseo para las innovaciones.

- Desarrolla la comprensión de medios ricos en contenido e induce a develar sus características.

- Permite desarrollar la habilidad para interpretar y comprender distintos tipos de cartografía.

- Mejora las relaciones con la comunidad, puesto que esta se considera como un laboratorio donde se obtienen muy buenos resultados.

- Promueve el desarrollo personal y permite el desarrollo de actitudes (Thralls, 1965, citado por Sánchez Ogallar, 1996)". 
El uso de la salida de campo en los proyectos de enseñanza de las ciencias sociales y las humanidades está confirmada por distintos autores. Hissa (2004, citado por Sánchez Ogallar, 1996); por ejemplo, enfatiza que independientemente de los objetivos que pueden justificarlo, este método puede ser útil en las prácticas de enseñanza por tratarse de una "posibilidad de comprender distintos lugares y paisajes, debiendo ser acompañado de referencias teóricas para que se constituya en un instrumento indispensable para la ampliación de las perspectivas conceptuales de los estudiantes".

La salida de campo se introdujo en la investigación geográfica con los viajes exploratorios realizados por científicos, muchos de ellos aventureros y pioneros, con los cuales pretendían recoger información que contribuyera en la sistematización de amplios campos de estudio y el acercamiento a los contextos reales.

Para Hissa, los trabajos de campo se caracterizan, porque se parte unas descripciones iniciales que progresivamente logran mayor consistencia, de modo que de relatos muy simples surgen amplios campos de interpretación; las descripciones producidas durante la observación en el campo se pueden incorporar, poco a poco, al desarrollo de los contenidos conceptuales y a los referentes escritos del objeto de referencia.- Los trabajos de campo de los cuales se originan descripciones e interpretaciones, cuando se refieren a las imágenes, serán paulatinamente capaces de servirse de una teoría de la imagen apropiada para el desarrollo de las ciencias sociales; los trabajos de campo, así como las experiencias interpretativas dadas durante su desarrollo se pueden enriquecer con conceptos teóricos y otros correlatos como los obtenidos mediante encuestas y entrevistas (Hissa, 2004, citado por Sánchez Ogallar, 1996).

Estas cuatro consideraciones son la base teóricometodológica de las salidas de campo como instrumento pedagógico. Este se convierte, así, en suma, en un método para desarrollar la capacidad de los estudiantes para identificar los discursos de las ciencias sociales. Pero la concepción de salida de campo puede cambiar según cambie la corriente teórica y metodológica del docente.

Por ejemplo, en la observación descriptiva y empírica, tan característica del positivismo, se busca entender el significado del fenómeno como algo externo. Es decir, esta práctica promueve un tipo de reconocimiento y observación desde un sujeto externo, capaz de capturar la información, analizarla y sistematizarla manteniéndose en un campo neutro frente a los fenómenos (Suertegaray, 2002).

Por otra parte, desde la óptica del materialismo histórico y dialéctico, el campo no es nunca externo al sujeto, es una extensión resultante de la interacción dialéctica entre el sujeto y el objeto. En este sentido, la práctica de la salida de campo se constituiría en un método que posibilitaría acceder al conocimiento a través de la vivencia en transformación; en otros términos, es una acción de observación de la realidad del otro, interpretada por la lente del sujeto en la relación con el otro sujeto. En la comprensión hermenéutica de la realidad, el campo es la expresión de las diferentes lecturas diferentes (Suertegaray, 2002). 
Desde cualquiera de estas dos perspectivas, el campo es el reflejo de nuestro espacio de vida. Es un espacio lleno de símbolos y significados siendo nosotros sus lectores. Por consiguiente, realizar una salida de campo, refleja sobre todo, una opción metodológica que responde a nuestras concepciones de mundo, a nuestras costumbres y tradiciones.

La salida de campo es un recurso didáctico válido para todas las disciplinas. No sólo se debe atribuir a los trabajos que buscan reflejar dinámicas espaciales con énfasis por los elementos físicos sino fundamentalmente humanos, es decir, sociales, políticos, económicos y culturales.

\subsection{La salida de campo: nuevas formas de enseñar y aprender}

En el nuevo marco de la sociedad del conocimiento, la cibercultura y los entornos virtuales de aprendizaje surgen como un nuevo concepto de espacio. Las organizaciones comerciales y todo tipo de instituciones se abren espacios a través de las redes y las comunidades electrónicas. En la enseñanza de las Ciencias Sociales y Humanas emergen a su vez métodos y tendencias que instauran nuevos caminos para el conocimiento y la comprensión de lo espacial.

Desde este punto de vista, la salida de campo se constituye en un marco referencial de trabajo muy amplio, en la medida que éstas permiten contrastar los elementos teóricos analizados en el aula de clase y referenciados a través de un texto impreso o digital con los escenarios geográficos reales.
Como lo expone Delgado (1988), "el trabajo de campo es una actividad científica asimilable metodológicamente a cualquier tipo de investigación". Toda salida de campo requiere de "un registro organizado de observaciones hechas en el campo dentro de una matriz espacial y un área de investigación definida previamente" (Delgado, 1988:7).

Desde el momento en el cual se elige el lugar que se va a visitar, empiezan a construirse una serie de imágenes mentales que son expresadas a través de palabras o signos. Así mismo, se construyen nociones y conceptos que luego son contrastados con las evidencias comunes, de carácter social, y personales, derivadas de la idiosincrasia individual. Por ejemplo, el término valle evoca una imagen mental común, pero cada persona encontrará un referente particular en relación con sus propias vivencias.

Deahílaimportanciadefomentarlapercepción directa de los elementos geográficos, de los entes institucionales, de las organizaciones sociales, de las agrupaciones humanas; de modo que se enriquezca el campo cognitivo de los estudiantes. En este aspecto, cabe distinguir tres niveles de entidades: lo físico -hechos, cosas, propiedades-, lo lingüístico -términos y frases- y lo conceptual -conceptos y proposiciones- (Bunge, 1969 citado por Sánchez Ogallar, 1996: 164).

Entre estos tres niveles se dan relaciones de designación (entre lo lingüístico y lo conceptual) y de referencia (entre lo lingüístico y lo físico ó entre lo conceptual y lo físico) de tal forma que al leer, por ejemplo, el término valle en un texto de geografía o los términos 
asentamiento y entidad; relacionamos por designación dicho término con la imagen mental, con la abstracción que designa. Sin embargo cuando percibimos sensorialmente un valle, una empresa, una iglesia, una edificación o una zona industrial, de manera concreta, establecemos una referencia entre lo físico y lo conceptual (Sánchez Ogallar, 1996:164).

Al evaluar la salida podemos asegurar que los estudiantes aprendieron significativamente una gran cantidad de conceptos y categorías que pueden poner en práctica y enseñarlos en un futuro como profesionales en distintas áreas. Estos conceptos, tal y como lo señala Sánchez Ogallar (1996: 165 citando a Graves, 1985), se pueden diferenciar en conceptos por observación, como cordillera, cabecera municipal, zona industrial, zonas de explotación agrícola o minera, zona turística, etc., y conceptos por definición tales como escala, latitud, longitud, altura sobre el nivel del mar, etc.

Así mismo los conceptos susceptibles de observación pueden ser: descriptivos simples, observables y accesibles (zona industrial); descriptivos con mayor dificultad de captación, inaccesible (zonas agrícolas, determinados productos) o por incluir varios conceptos como producción mixta (agrícola e industrial); descriptivos muy complejos, donde se requiere plena comprensión previa de múltiples conceptos relacionados, por ejemplo, como el caso de una formación geológica determinada para la explotación de cemento ó una zona de producción lechera.

Por otra parte, los conceptos por definición pueden ser simples cuando representan las relaciones entre dos variables como densidad de población, índice de natalidad, aforo, etc.; y complejos, cuando se forman por las relaciones complejas entre dos o más variables, como una determinada producción económica ó una interpolación estadística.

Gracias a procesos intelectuales basados en la observación y en la intuición, el trabajo de campo en geografía y en el ámbito de las ciencias sociales y humanas permite la observación de hechos que funcionan como referentes del proceso de análisis, especialmente en los conceptos descriptivos de mayor complejidad.. A partir de estos conceptos se plantean núcleos problemáticos, entendidos como ejes provenientes de la identificación de necesidades, polos de interés que demandan estudio, comprensión e intervención en forma inmediata ó a mediano plazo. 


\section{LAS SALIDAS DE CAMPO EN LA FORMACIÓN DE EMPRENDEDORES- ANÁLISIS DE LOS DOCENTES}

Cada salida de campo está acompañada de un proceso de sistematización y análisis donde se relacionan los lugares visitados, los recorridos, los tiempos y los fenómenos institucionales y organizacionales encontrados en cada itinerario. Para el caso específico de este trabajo y mediante la metodología del focus group, se buscó conocer y comprender la visión que tienen los profesores que realizan salidas de campo como estrategia pedagógica en la formación de emprendedores y su influencia en la vida personal y profesional.

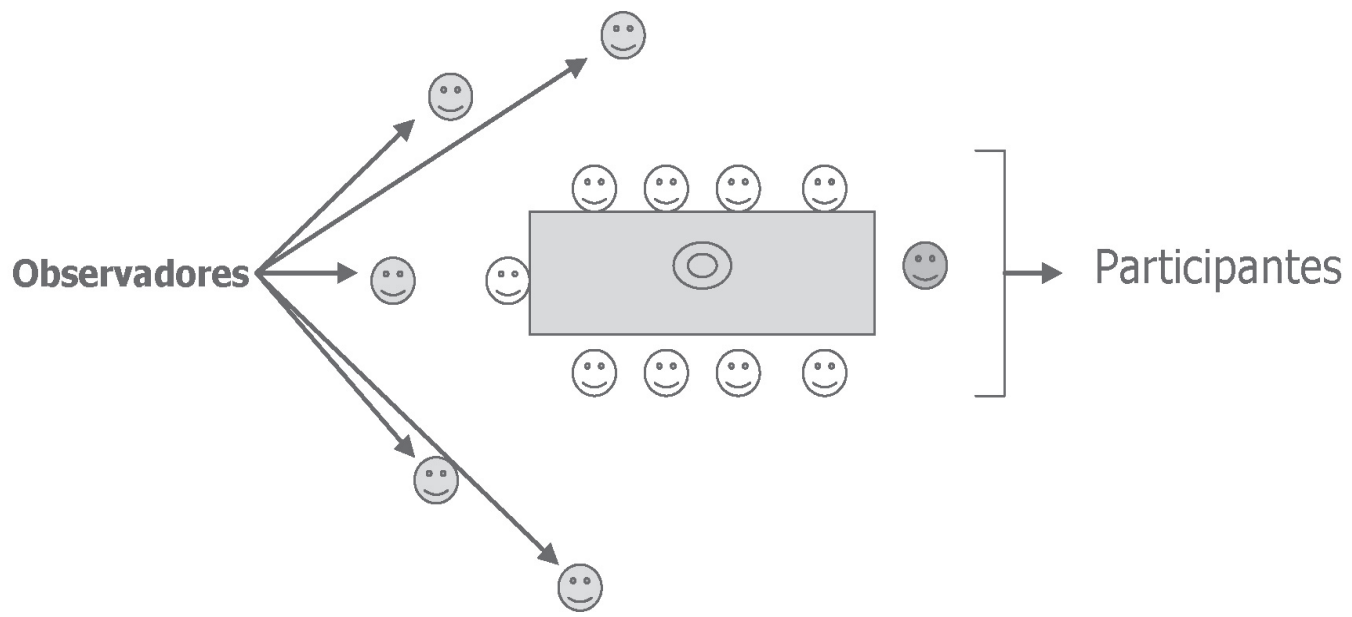

Los participantes discutieron entorno a los siguientes interrogantes:

- ¿Cuándo considera importante recurrir a las salidas de campo cómo ambiente de aprendizaje y cómo estrategia didáctica?

- ¿Cuál es su criterio para escoger los lugares que se visitan en la salida de campo?

- ¿Cómo responden la salida de campo y su metodología, a las competencias propuestas por la Universidad y en particular al desarrollo del espíritu emprendedor?

- ¿Cómo evalúa y retroalimenta la salida de campo?

\subsection{Análisis de resultados}

Históricamente los profesores han encontrado en la salida de campo una estrategia pedagógica que contribuye a la formación de emprendedores. Porunlado, esuna estrategia que involucra una actitud emprendedora desde su misma preparación y gestión; demanda además, una capacidad operativa instintiva por parte de todos sus participantes; por otro, enfrenta a los estudiantes con los espacios reales y los ámbitos de propios de las empresas y las organizaciones en amplios marcos culturales, en suma, es un conjunto de saberes concretos que afianzan las competencias sociales y culturales de estudiantes y profesores. 
5.1.1 ¿Cuándo considera importante recurrir a las salidas de campo como ambiente de aprendizaje y cómo estrategia didáctica?

Las salidas de campo como propuesta pedagógica sustentan su metodología en la creación de ambientes y experiencias que afiancen el compromiso y el conocimiento de los estudiantes. La preocupación del profesor es generar los espacios motivadores e interesantes para el aprendizaje, con el fin de determinar variables y campos de intervención en la sociedad.
Uno de los objetivos centrales de las salidas de campo, de acuerdo con los docentes, es el de propiciar diferentes lecturas del espacio y de las situaciones sociales. En el caso de las carreras sociales, técnicas y científicas; las salidas pueden ser el origen de propuestas, de respuestas objetivas en términos profesionales $y$ en un futuro cercano, a los problemas de la sociedad. Por otro lado, es importante para los docentes contar con información concreta para su trabajo en el aula. A continuación se presenta una gráfica conceptual de la interpretación y de las opiniones de los profesores.

\section{Esquema 1}

Objetivos en utilizar la salida de campo como ambiente de aprendizaje

¿Cuáles son sus objetivos cuando utiliza la salida de campo como AMBIENTE (aula, patio, parque, estadio, sala, recorridos urbanos) de aprendizaje?

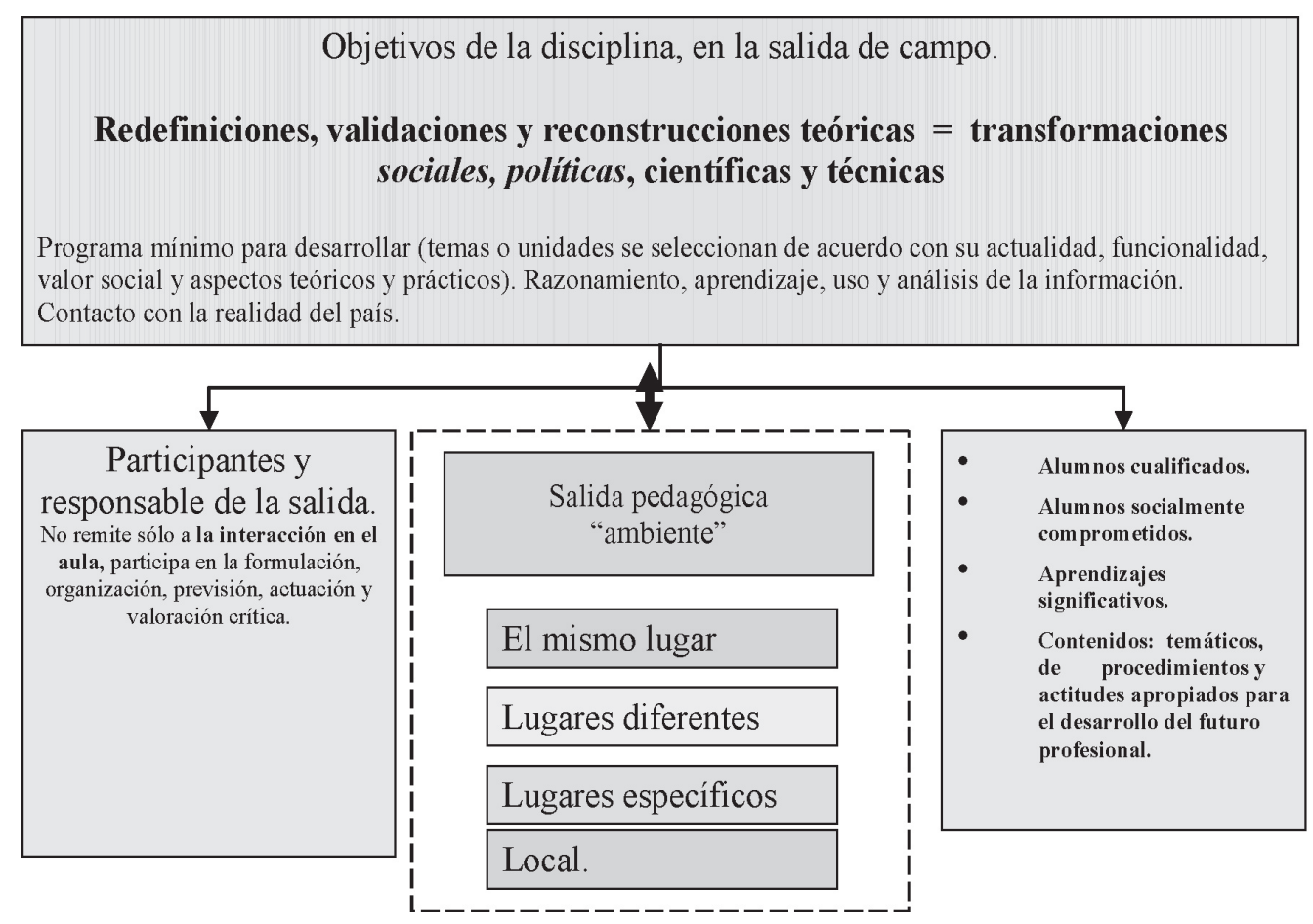




\subsection{2 ¿Cuál es su criterio para escoger los lugares que se visitan en la salida de campo?}

Dentro del proceso de enseñanza-aprendizaje, muchos conceptos se utilizan con cierta flexibilidad, pero es preciso que la salida de campo como estrategia pedagógica tenga una estructura coherente, que incluya una serie de acciones lógicas y cuente con materiales de referencia claros. Es igualmente necesario contar con los intereses del estudiante y con su nivel formativo. La selección del lugar depende también de las necesidades de la asignatura y de los ejes problémicos propuestos a lo largo del curso, así como de los mismos proyectos de aula que hayan dado lugar a las discusiones iniciales con los estudiantes.

El profesor no puede concebirse como una enciclopedia o guía, sino como un intelectual, que comprende la lógica de la estructura de su ciencia y que entiende de forma histórica y evolutiva los procesos y vicisitudes de su formación como disciplina desarrollada por una comunidad científica.

Bajo estas características los alumnos visitan ambientes naturales o instituciones donde observan y recogen datos; tanto para examinar comportamientos o relaciones como para responder a sus propios intereses y obtener puntos de vista, que permitan consolidar la formación académica, la misión y visión de la Universidad y adquirir un compromiso con la nación en términos profesionales.

En la exploración y salida pedagógica el alumno visita o busca lugares en los que pueda observar y describir acontecimientos que considere importantes. No sólo aprenden los alumnos en el aula de la universidad, también en el campo, en el lugar del hecho, en contacto directo con las organizaciones y los sectores de producción. Una salida puede ser una excursión educativa, una experiencia de campo, una exploración (de campo), una observación en el terreno, independiente o supervisada.

Las salidas pueden ser locales o lejanas; pueden incluir estadías y estar orientadas a un área profesional; pueden incluir encuestas de opinión en los mercados, es decir, actividades académicas y extra académicas. En todos estos casos, los alumnos aprenden observando y descubriendo en contacto directo con la realidad. Uno más de los criterios que inciden en la selección del lugar está dado por los conocimientos previos o por los aspectos que han sido analizados en clase. A menudo se observa, también, lo inesperado o desconocido.

Se pueden resumir los aspectos más relevantes de esta actividad de la siguiente manera:

- La importancia de la motivación como factor determinante en el proceso de enseñanzaaprendizaje.

- La pertinencia de la salida para el desarrollo de la asignatura y su correspondiente programa académico.

- El mayor aprovechamiento de la experiencia profesional del docente.

- La atención a los gustos y pasiones personales por parte del docente y del alumno. 
- La posibilidad de realizar contactos personales como oferta de visita.

- El desarrollo paralelo de procesos de investigación y experimentación.

- La retroalimentación de la información recogida en la salida en actividades de clase.

- El protagonismo del alumno como agente de su propio aprendizaje.

- La construcción del aprendizaje desde la realidad actual de cada alumno y del programa que estudia.

- El afianzamiento en las técnicas de trabajo intentando asentar hábitos de constancia y responsabilidad profesional.

- La propagación de las capacidades de análisis crítico, el discernimiento y la toma de decisiones.

- El desarrollo de tareas en grupo con actitud participativa.

- La valoración de actividades culturales, emprendedoras, complementarias.

- La negociación encaminada al crecimiento del alumno en sus decisiones personales, profesionales y sociales, potenciando los valores de servicio ético y la responsabilidad social.

- El desarrollo de ambiente propicio para el aprovechamiento académico, cuidando especialmente las relaciones entre compañeros y con los profesores, así como el respeto a los lugares, la idiosincrasia, la cultura y las instalaciones.

- El cumplimiento de las normas de convivencia, valorando la disciplina y la puntualidad como medios eficaces para el rendimiento.

- La colaboración y la divulgación entre todos los que componen la comunidad educativa, asumiendo cada uno su responsabilidad en el objetivo común.

\subsection{Criterios espaciales}

La salida pedagógica se debe orientar por las sensaciones que genera el lugar, por las situaciones o institución que se encontrarán en el itinerario de acuerdo con el plan de observación. El espacio, se dispone como medio de aprendizaje generador de experiencias diferentes a las transmitidas en forma oral y figurada en el espacio académico. En la salida se da lugar a innumerables situaciones imprevistas de aprendizaje, que hacen parte de las ventajas adicionales de esta metodología. Tales experiencias en relación con el espacio, pueden derivar de algunos de los siguientes factores:

En cuanto a la logística de forma en la salida.

- Limitaciones (programas de los estudiantes, género y edad).

- Participantes, número de grupos o personas.

- Guías. 
- Experiencias y reflexiones personales.

- Fuera y dentro de la ciudad.

- Escenarios rurales y urbanos, equipamientos públicos y privados.

- Criterios específicos de la salida en cuanto a manual de convivencia estudiantil.

En cuanto al fondo mismo de la salida.

- Empresas e instituciones a visitar (contactos, personas que recibirán el grupo, objetivos de la visita a cada institución, posibles recorridos e intereses específicos).

- Instrumentos de apoyo a la obtención de información (encuestas, formularios de entrevista, ruta fotográfica y guión de video, rutas de observación, criterios de descripción, análisis reflexivos).

- Apoyo al programa ó asignatura. Ser claros y concisos para qué visita tal o cual lugar.

- Procesos de socialización de la salida (conferencias, videos, exposiciones fotográficas, producción de escritos, etc).

- Evaluación de la salida en cuanto a lo actitudinal, procedimental y conceptual.

\section{Etapas}

Se definen tres etapas para tener en cuenta dependiendo del lugar visitado:

- Planteamiento. En esta etapa se formulan los objetivos académicos y los planes de trabajo adaptados a los objetivos previstos. La formulación de un plan implica la toma de decisiones anticipada y la reflexión con anterioridad a la puesta en operación de la salida de campo. Siempre será necesario prever situaciones alternativas, el denominado Plan B, como posibilidad por si algún elemento llega a fallar. Ejemplo: alternativas de alojamiento o de alimentación, dado el caso.

- Ejecución. Posteriormente al planteamiento, el profesor pone en práctica los recursos y métodos didácticos afines al proceso de enseñanza.

- Evaluación. Es la etapa en la cual se verifican los resultados obtenidos con la ejecución, materializándose en el proceso de evaluación y donde se retroalimenta la salida. 


\section{Esquema 2}

Criterios para escoger el o los lugares que se visitan en la salida de campo ¿Cuál es su criterio para escoger los lugares que se visitan en la salida de campo?

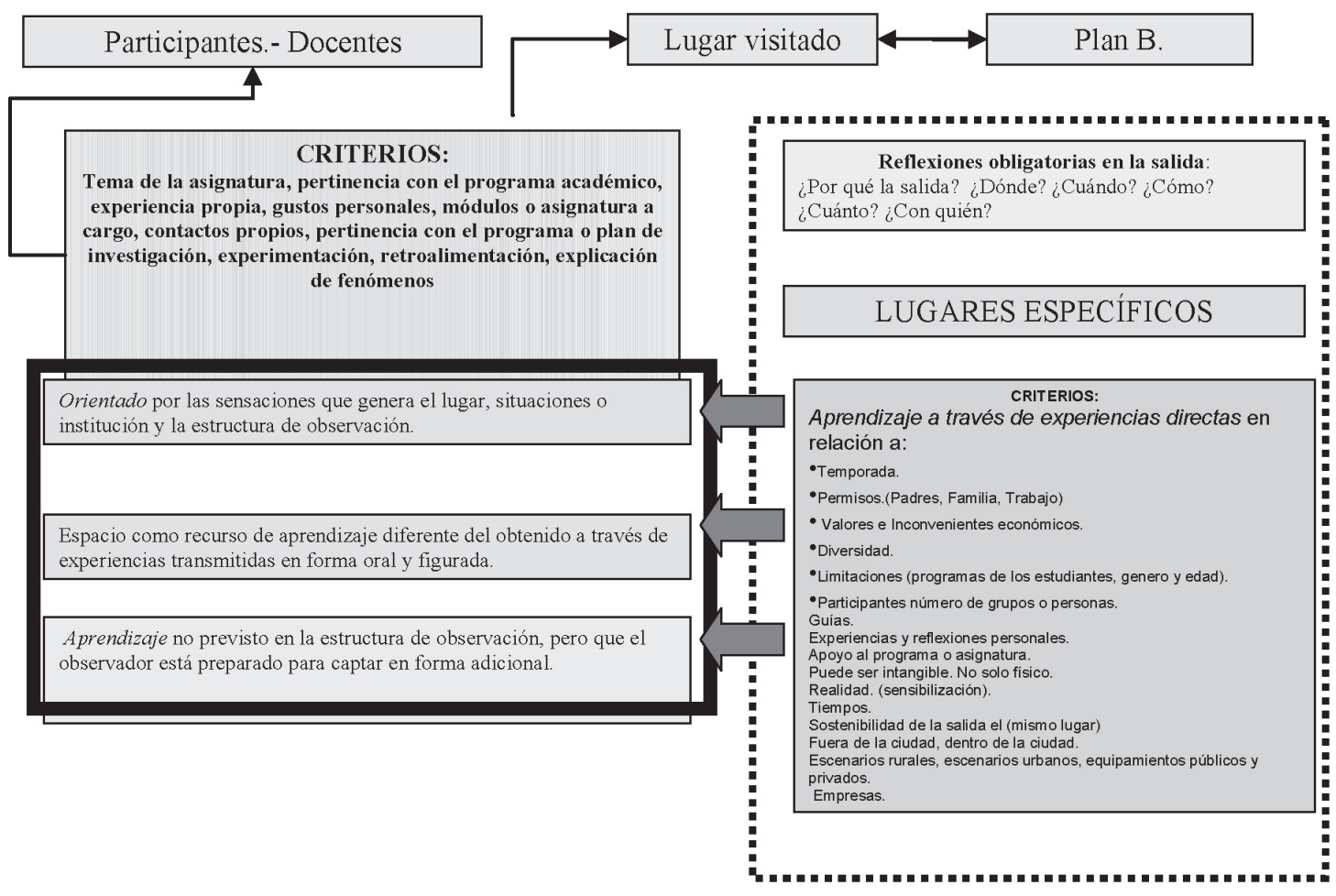

ANÁLISIS DE LA SITUACIÓN

Situación de partida: restricciones de los medios, material, e infraestructura, nivel de formación del docente, tiempo.

Expectativas y conocimientos previos de los alumnos, nivel de madurez e intelectual, intencionalidades formativas, aspiraciones de desempeño futuro $=$ prueba de entrada.

EN SINTESIS: limitaciones de tiempo, espacio, medios y personal.

\section{¿CÓMO RESPONDE LAS SALIDAS DE CAMPO A LAS COMPETENCIAS PRO-
PUESTAS POR LA UNIVERSIDAD DE MANERA ESPECIAL A LA COMPETENCIA EMPRENDEDORA?}

El aprendizaje en las salidas pedagógicas constituye una actividad mental del sujeto que aprende, recuerda, transmite, participa; permitiéndolelaadquisicióndeconocimientos, hábitos y actitudes, así como la retención y utilización de los mismos; originando una modificación de la conducta y un estrecho vínculo social, profesional, e intelectual. En este sentido, las competencias desarrolladas en las salidas de campo definen actividades de aprendizaje y generan secuencia de acciones encaminadas a la construcción del conocimiento, al desarrollo de habilidades y a la formación de actitudes. 
La profundidad y la calidad del aprendizaje estarán determinadas por el conocimiento y comprensión de la naturaleza de la salida pedagógica, en términos de las competencias sociohumanística, emprendedoras, comunicativas, tecnológicas, investigativas y por la información que se posee sobre el tema, así mismo, por el grado de control que se ejerce sobre los procesos cognitivos implicados: atención, memoria, razonamiento, interpretativas, propositivas y argumentativas.

Se trata de relacionar, descubrir, fortalecer, contextualizar, interactuar con el espacio a visitar con un problema, lo que el alumno ya sabe de una forma organizada y no de un modo arbitrario.

Para que se produzca el aprendizaje y que las competencias se cumplan con base en el proyecto educativo institucional, se precisa coherencia en la estructura interna, material y secuencia lógica en el desarrollo de la salida.

Además, los contenidos deben ser comprensibles desde la estructura cognitiva que posee el participante (alumno) que aprende. Para que la salida de campo perdure en el que participe, la eficacia de las competencias se sitúa en la calidad de las estructuras internas, de los esquemas de pensamiento y actuación que desarrolla el docente, el lugar a visitar y el participante (alumno), no en asociaciones pasajeras.

Por tanto, las competencias a desarrollar, el emprendimiento y el aprendizaje son fenómenos correlativos y relacionados por lo que se denomina la relación didáctica y pedagógica de la salida de campo.

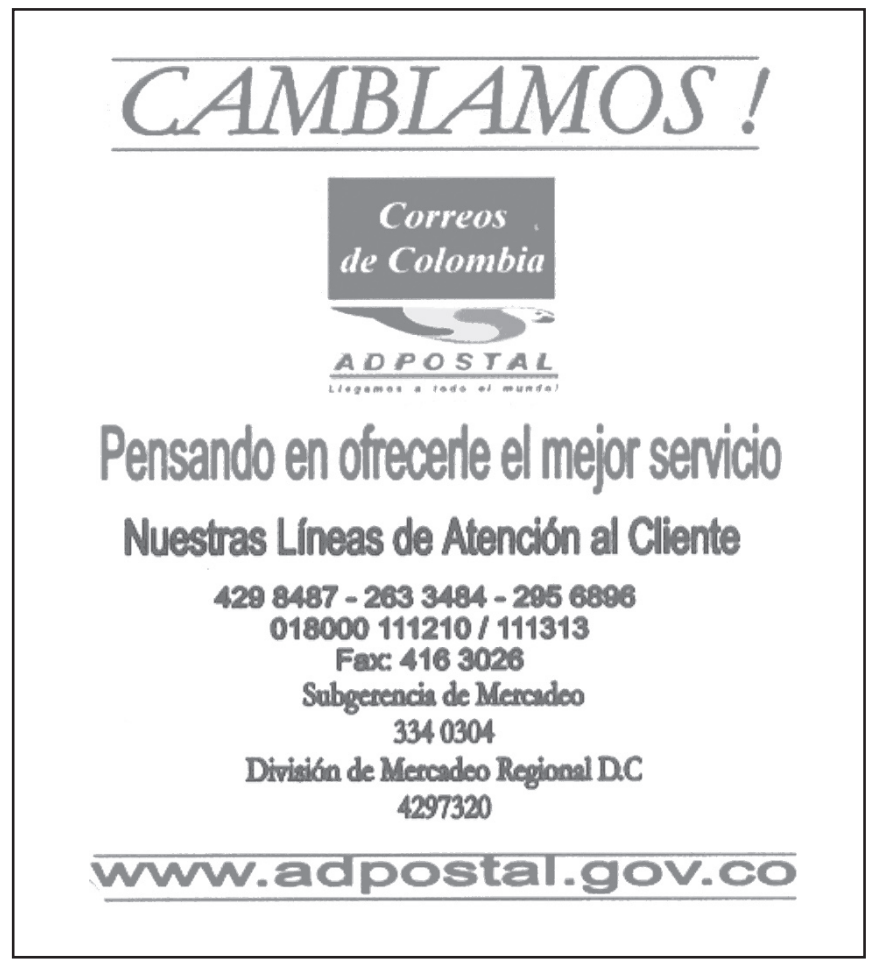


Esquema 3

La salida de campo como responde a las competencias propuestas por la universidad y de manera especial a la competencia emprendedora

¿La salida de campo cómo responde a las competencias propuestas por la universidad EAN y de manera especial a la competencia emprendedora?

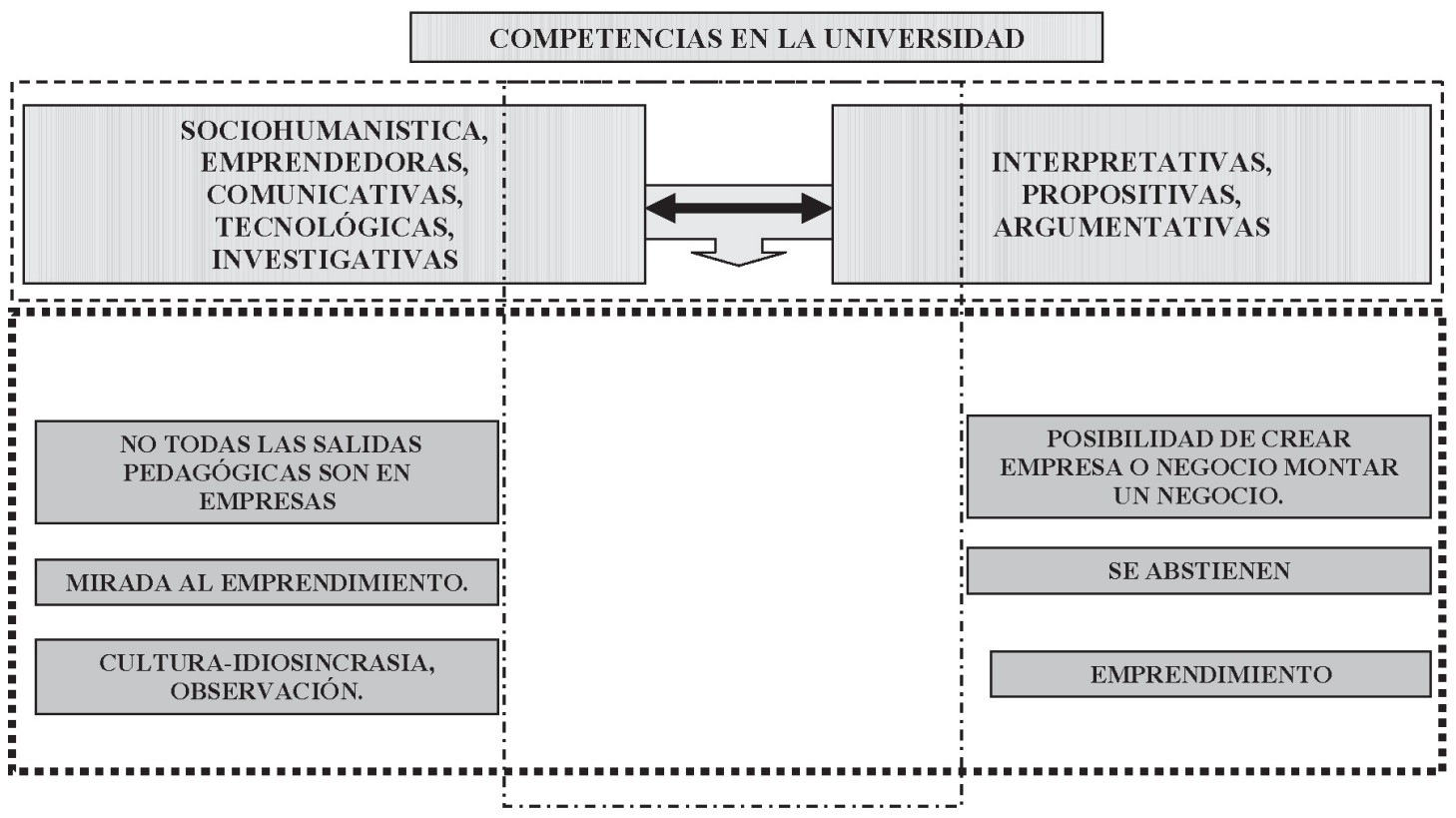

El proceso de evaluación permite verificar el cumplimiento de los objetivos educativos y comprobar que se ha producido el aprendizaje previsto en la salida pedagógica. Se tratapor tanto, de evaluar lo que el alumno ha aprendido durante de la fase de enseñanza. Es preciso distinguir los términos verificación y evaluación del aprendizaje. Definimos verificación como el proceso por el cual se comprueba lo aprendido por el alumno en la salida; mientras que evaluación, es el proceso de atribución de valor que se traduce en una calificación o una nota, aunque en las salidas no siempre se califica. Los objetivos de los sistemas de evaluación del aprendizaje de los alumnos en la salida pedagógica son entre otros: servir para valorar el grado de cumplimiento de los objetivos educativos, diagnosticar errores conceptuales, habilidades o actitudes de los alumnos para analizar las causas de un aprendizaje deficiente y tomar las medidas correctivas oportunas. 
Mediante la evaluación se valoran los resultados obtenidos en el proceso educativo. Evaluación es el proceso de obtención de información de diferentes niveles y de su uso para formular juicios que se utilizan para tomar decisiones y traducirlos en reflexiones compartidas con el estudiante.

En el proceso de evaluación es fundamental analizar aspectos como:

- La calidad de la reflexión a la que la salida ha dado lugar.

- Los niveles de observación y el tipo de registro que se ha alcanzado.

- La profundidad del análisis de las situaciones observadas.

- El espíritu crítico y la mirada sobre la realidad.

- La capacidad de adaptarse a nuevas situaciones y de resolver eventos circunstanciales e imprevistos.

La retroalimentación de la salida se lleva a cabo en tres momentos:

- Preparación. Se trata en esta fase de describir la información necesaria y de construir los instrumentos de recolección de información. Preparar y elaborar las pruebas que se realizarán durante la salida; por su parte los alumnos deben definir las muestras finales y los criterios para su evaluación, es decir, el producto final.

- Obtención de la información. Supone la realización de ejercicios orales y escritos: ensayos, trabajos finales y otras formas de registro; en algunos casos, productos tangibles como fotografías para exposiciones, juegos de divulgación; incluso, actividades de largo alcance como seminarios, planes de negocios o convenios institucionales.

- Toma de decisiones. Consiste enformular juicios, tomar decisiones, resumir y dar a conocer la evaluación, con respecto a los tipos de sistemas de evaluación.

Para retroalimentar las salidas de campo se identifican diferentes resultados directamente asociados a una respuesta, reflexión o documentos de mejoras para futuras salidas con base en la evaluación dada por los estudiantes en la salida, los resultados abren la posibilidad de realizar ajustes en este aspecto como un manejo más estricto en la planeación y el planteamiento de una o dos actividades como plan de contingencia.

El desarrollo de la actividad pedagógica, es la parte central y por lo tanto donde los estudiantes deben tener plena conciencia de los objetivos que se pretenden alcanzar con la salida a fin de desarrollar plenamente las competencias, de allí que se propone la realización de un taller durante los recorridos y un tiempo de retroalimentación para atraer nuevamente la concentración de los estudiantes.

Los resultados de la salida y el desarrollo de las competencias pedagógicas van ligadas directamente a una buena puesta en operación de la actividad realizada en el espacio a visitar, la planeación y las expectativas personales que se habían planteado los estudiantes, el docente y el "espacio visitado, empresa etc.", por tal 
razón es significativo realizar una retroalimentación inmediata por medio de una plenaria en las que se puedan filtrar y poner en la mesa las inquietudes y ajustes de la salida de campo y las mejoras a los punto anteriores porque esto lleva a un beneficio múltiple debido a que aumenta todos los indicadores.

\section{Esquema 4}

¿Qué criterios de evaluación y cómo retroalimenta la salida de campo?

\section{¿Qué criterios de evaluación y cómo retroalimenta la salida de campo?}

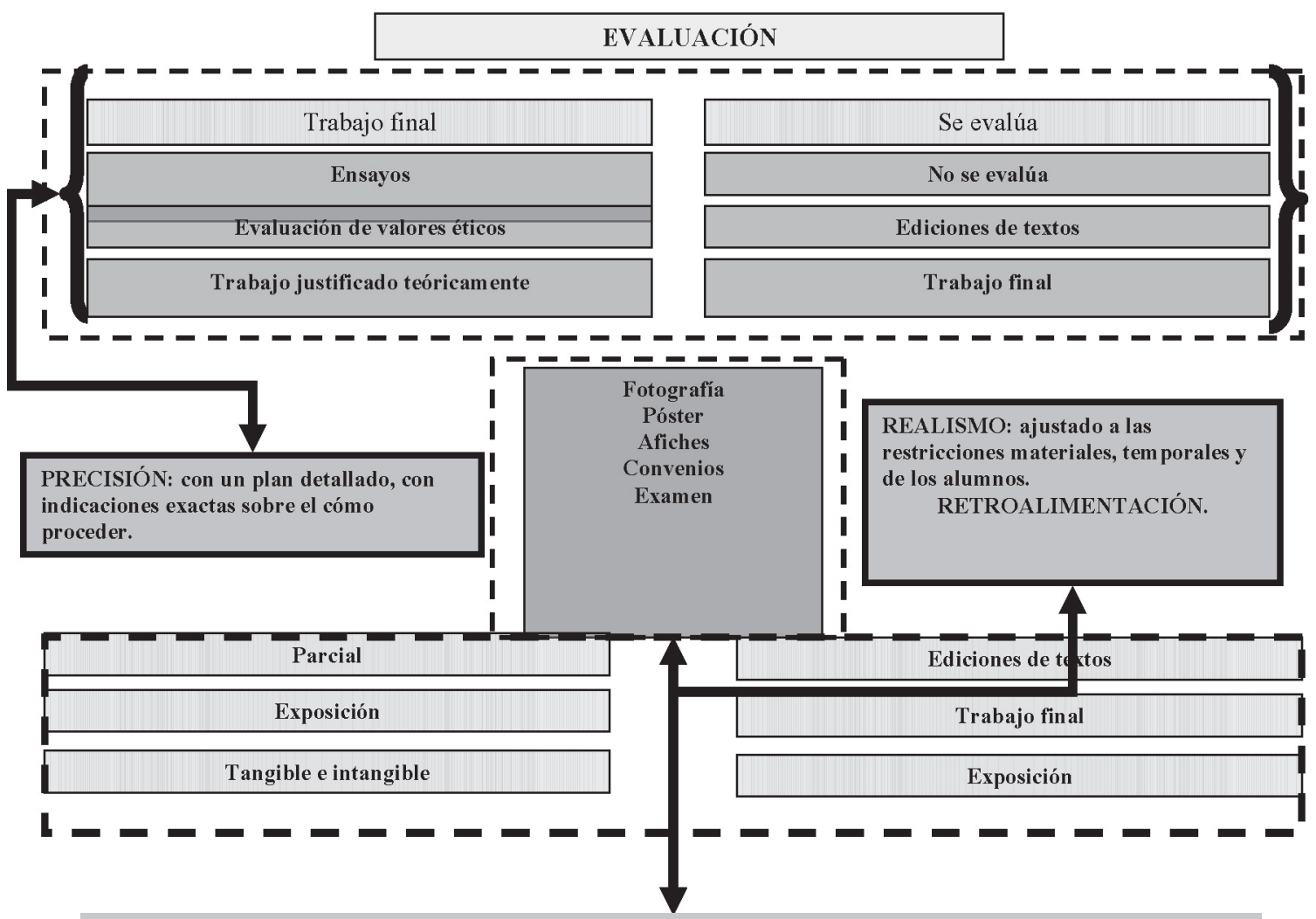

FLEXIBILIDAD: posibilidades de adaptación de la salida con los criterios de evaluación 
La salida de campo como estrategia pedagógicaenlaformacióndeemprendedores brinda distintas posibilidades de acción para los maestros y los estudiantes, en tal sentido referimos una de las vivencias desarrolladas en el marco escolar universitario, como un testimonio gratificante en el estudio de distintos lugares, que posibilitarían su análisis para la creación de planes de negocios.

Las salidas de campo permiten a los estudiantes y profesores, en este caso como observadores, analizarlasmúltiplesdinámicas que se presentan como oportunidades de negocio. El visitar distintos lugares, adquiere sentidograciasal permanenteestablecimiento de nexos, al desempeño de funciones y al significado de los distintos actores sociales que se involucran en el proceso.
El realizar la salida de campo también posibilita involucrar sujetos que mediante diversas intenciones descubren distintas caras y distintos lugares en donde surgen múltiples facetas culturales, que a la postre revelan una sociedad que es vista a través de su gente, sus edificios, sus calles y carreras; sus parques, sus empresas e instituciones, su monumentos, sus sonidos, etc. Así mismo permite a todos los que en ella participan reconocerse como actores protagónicos de esos escenarios que a la postre permitirán ampliar su nivel de reflexión sobre lo que es el país y lo que él requiere.

La salida de campo es una estrategia válida para conocer la realidad desde la observación, análisis, descripción y reflexión del quien conoce y experimenta el lugar, en este caso profesores y estudiantes emprendedores. 


\section{Bibliografía}

Argüelles, D; Nagles, N (2006). Estrategias para promover procesos de aprendizaje autónomo. Bogotá D.C: Universidad EAN.

Ausubel, D (1970). La teoría del aprendizaje significativo. En: http://www.wikilearning.com/ tutorial/teorias_del_aprendizaje-ausubel_la_teoria_del_aprendizaje_significativo/12263-6).

Bruner, J (1970:1980). Investigaciones sobre el desarrollo cognitivo. Madrid: Pablo del Río.

Cely, A y Lache, N (2006). La salida de campo: Una experiencia significativa en el aprendizaje de la geografía. En: Lupa Geopedagógica. No. 1. Bogotá D.C.: Universidad Pedagógica Nacional, p.p. $4-6$.

Cely, A y Díaz N. (2005). Procesos de aprendizaje de las competencias sociohumanísticas en la Escuela de Administración de Negocios EAN. Informe Final de Investigación. Bogotá D.C: Universidad EAN.

Coll, C (1991). Aprendizaje escolar y construcción del conocimiento. Madrid: Paidós.

Coll, C. et al., (2002). El constructivismo en el aula. Barcelona: Grao.

Delgado, O (1988). Indicaciones metodológicas para el trabajo de campo en geografía. Cuaderno No. 1. Bogotá. D.C: Universidad Pedagógica Nacional.

Flórez, R (1994). Hacia una pedagogía del conocimiento. Bogotá. D.C: Mc Graw Hill.

Flórez, R (1999). Evaluación, Pedagogía y Cognición. Bogotá. D.C: Mc Graw Hill.

Lafrancesco, G (1998). El papel del currículo y sus planificadores. La gestión curricular, problemáticas y perspectivas. Bogotá D.C: Editorial Libros y Libros.

REVISTAEAN (2003). Modelos pedagógicos en el desarrollo de competencias emprendedoras e investigativas.. Bogotá D.C.: EAN - Centro de Investigaciones. No. 48, mayo-agosto de 2003.

Sánchez, A (1996). El trabajo de campo y las excursiones. En: Enseñar geografía de la teoría a la práctica. Madrid: Síntesis, p.p. 159-184. 
Sierra, G(2003). Una aproximación pedagógica para formar competencias. En: Revista Escuela de Administración de Negocios EAN.. Bogotá D.C: No. 48, Mayo agosto de 2003, p.p. 28-39

Silva, J (2004). Cómo iniciar su propio negocio. El perfil del empresario. Bogotá: Escuela de Administración de Negocios EAN.

Souto, X (1999). Didáctica de la geografía. Barcelona: Ediciones del Serbal.

Suertegaray, M (2002). Geografia, transformacoes sociais e engajamento professional: o trabalho do geógrafo no Brasil. Revista Scripta Nova: Revista Electrónica de Geografía y Ciencias Sociales, No. 119.

UMAÑA, G (2004): Importancia de las salidas de Campo en la Enseñanza de la Geografía. En: Revista Folios Segunda Época. Bogotá D.C: No. 20, Segundo Semestre de 2004, Universidad Pedagógica Nacional, p.p. 105-120.

http://www.monografias.com/trabajos5/teorsist/teorsist.shtml\#retrp-. 